\title{
Evaluation of Compressive and Energy Absorption Characteristic of a Closed-cell Polymeric Foam subject to Dynamic Loading
}

\author{
Takahiro Kawano ${ }^{1}$, Yuta Takase $^{1}$, Tomohisa Kojima ${ }^{1 *}$, Hiroyuki Yamada ${ }^{2}$, \\ Kohei Tateyama ${ }^{3}$, and Tomoaki Tsuji ${ }^{1}$ \\ ${ }^{1}$ Chuo University, Dept. of Precis. Mech., 1-13-27, Kasuga, Bunkyo-ku, Tokyo, Japan \\ ${ }^{2}$ National Defense Academy, Dept. of Mech. Eng., 1-10-20, Hashirimizu, Yokosuka, Kanagawa, Japan \\ ${ }^{3}$ Ritsumeikan University, Dept. of Mech. Eng., 1-1-1, Nojihigashi, Kusatsu, Shiga, Japan
}

\begin{abstract}
Foamed plastics have been used in many engineering fields because of their superiority in low density, energy absorption, thermal insulation, and acoustic damping capacities. With foams, it is known that the microstructure of cells directly relates to macroscopic deformation behaviour. However, mechanical properties based on microstructures composed of non-uniform cells have not been fully understood. This study aims to clarify the mechanical properties grounded on microstructures of foamed plastics subjected to dynamic loading. The quasi-static and dynamic compression test was carried out using foamed plastic with anisotropy in the cell structure, then the strain rate dependence of deformation and energy absorption characteristics was investigated. It was confirmed that the local buckling of the cells was the dominant deformation mode in the plastic collapse of the test piece. It was also confirmed that cell buckling was initiated around the middle in the height after the plastic collapse, then propagated to the whole specimen in both the quasi-static and dynamic tests by using digital image correlation. The stress-strain relationships and the amount of absorbed energy showed strain rate dependence owing to the deformation mode in which the local buckling of the cells is dominant.
\end{abstract}

\section{Introduction}

Foamed plastics are used in many engineering fields such as packaging materials and building insulation materials, because of their small density and excellent mechanical energy absorption characteristics, thermal insulation, and acoustic damping properties [1-2]. As the deformation characteristics of foams, it is known that the deformation of microstructure composed of cells directly affects the whole deformation. Numerous studies have been carried out on the prediction of mechanical properties of foamed plastics concerning the microstructure both with low and high strain rate [3]. Gibson and Ashby proposed a theoretical model that predicted the characteristics of closed-cell forms in various deformation modes using simple unit cell models [1]. However, the actual foamed plastics

* Corresponding author: kojima.31k@g.chuo-u.ac.jp 
are an assembly of non-uniform cells. Thereby in recent years, studies have been carried out with inhomogeneous cell structures using finite element analysis [4-6]. Nevertheless, the relationship between the deformation characteristics of the non-uniform cell and the mechanical properties of the foamed plastic has not been clarified sufficiently. This study aims to clarify the relationship between the deformation characteristics of foamed plastics subjected to dynamic loading and deformation characteristics of cell structures. In this paper, the quasi-static and dynamic compression tests were carried out using foamed plastics with anisotropic cell structure to investigate the strain rate dependence of deformation and energy absorption characteristics.

\section{Experimental method}

The polypropylene foamed plastic (Zetron, Sekisui Chemical Co., Ltd.) was used as the test material. This foamed plastic has an anisotropic cell shape by controlling the foaming direction when it was manufactured. The expansion ratio and height differ depending on the degree of foaming. In this study, we used the test pieces with three kinds of expansion ratios. Table 1 shows the conditions of the test piece. Figure 1 shows the test piece with the expansion ratio of 12 as the representative.

Table 1. Geometric parameters of the material used in the study [4].

\begin{tabular}{|c|c|c|}
\hline Expansion ratio & Height [mm] & Relative density [\%] \\
\hline 10 & 5.5 & 6.5 \\
\hline 12 & 10 & 5.0 \\
\hline 15 & 12 & 4.0 \\
\hline
\end{tabular}

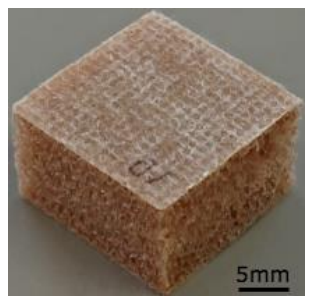

(a)

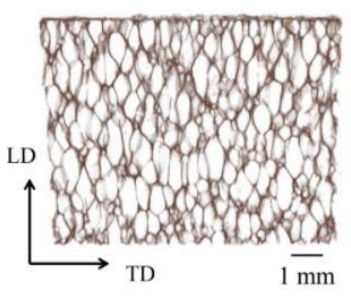

(b)

Fig. 1. Test piece (expansion ratio of 12); (a) Macroscopic view, (b) cross-section [4].

The quasi-static and dynamic compression tests were conducted to evaluate the compressive response. In the quasi-static test, the universal testing machine (AGS-J 100N, Shimadzu Corporation) was used with a test speed of $1 \mathrm{~mm} / \mathrm{min}$. The corresponding strain rates were 1.39-3.03 $\times 103 / \mathrm{s}$ depending on the height of the test piece presented in Table 1 . In the dynamic test, the drop-weight testing machine designed by Yamada et. al [7] was used with a test speed of $0.37 \mathrm{~m} / \mathrm{s}$. The corresponding strain rates were $16-35 / \mathrm{s}$ depending on the height of the test piece. The width and length of the test piece used for the quasi-static test were both $20 \mathrm{~mm}$ and for the dynamic test were $18 \mathrm{~mm}$.

The strain distribution of the test piece in the thickness direction was measured using digital image correlation (DIC) from the photographs taken during the tests. The software Ncorr v1.2 that operates using MATLAB was used for DIC [8]. The subset radius and the subset spacing were 30 and 5 pixels, respectively. Figure 2 shows the random pattern applied to the side surface of the test piece. In the photographs for DIC, the number of pixels for the 
height of the test piece was 462 and 480 pixels for the quasi-static and dynamic test, respectively. Therefore, it can be said that the images for DIC have a similar number of pixels in the region of the test piece.

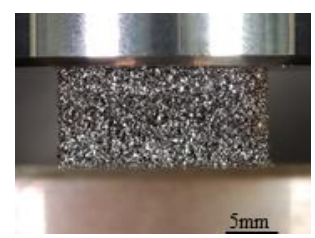

(a)

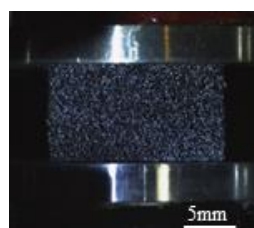

(b)

Fig. 2. The random pattern applied on the side of the test piece; (a) in the quasi-static test, (b) in the dynamic test.

Besides, the amount of absorbed energy was estimated from the stress-strain curve obtained by the tests. With the strain and the stress at the initiation of densification $\varepsilon_{\mathrm{p}}$ and $\sigma_{\mathrm{p}}$, the amount of absorbed energy $W$ can be expressed by the following equation.

$$
W=\int_{0}^{\varepsilon_{\mathrm{p}}} \sigma_{\mathrm{p}} d \varepsilon
$$

\section{Results and discussion}

As a typical example, the results of the test pieces with an expansion ratio of 12 are presented here. Figures 3 and 4 show the deformation of the test piece during the quasi-static and dynamic compression test, respectively. It can be confirmed that the side of the test piece was deflected outward by compression.

Figure 5 presented the stress-strain curves obtained by the quasi-static and dynamic compression tests of the test piece in the case of an expansion ratio of 12. The stress-strain curves have a peak after the elastic part. It indicates that the plastic collapse of the test piece was induced by local buckling of cells [9]. It was confirmed that the plastic collapse stress was increased with a higher strain rate. This may because the buckling of the cells was the dominant deformation mode in the plastic collapse of the test piece.

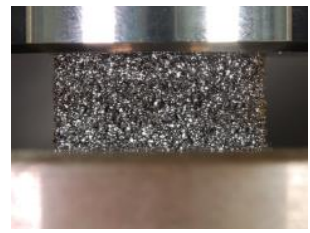

(a)

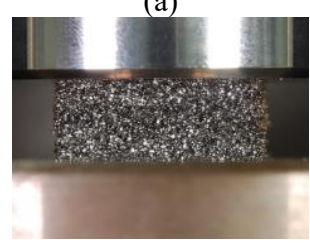

(d)

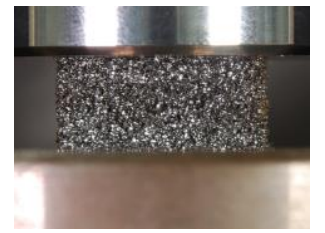

(b)

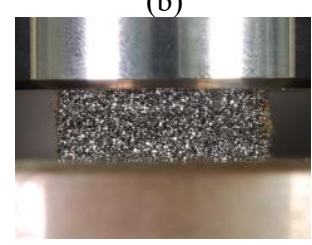

(e)

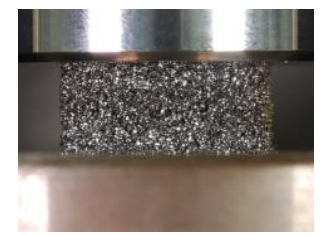

(c)

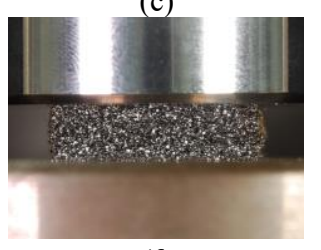

(f)

Fig. 3. Deformed test piece in quasi-static test in the case of expansion ratio of 12 ; at engineering strain of (a) 0.04 , (b) 0.07 , (c) 0.10 , (d) 0.20 , (e) 0.30 , (f) 0.45 . 


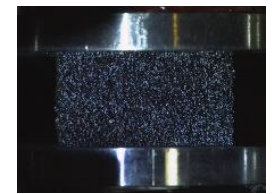

(a)

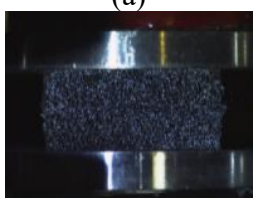

(d)

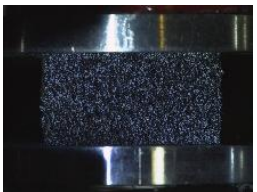

(b)

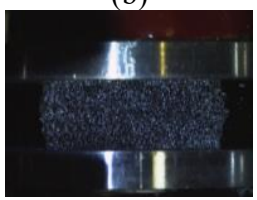

(e)

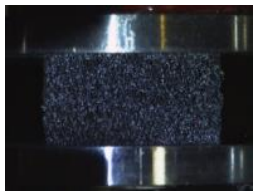

(c)

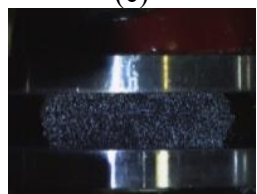

(f)

Fig. 4. Deformed test piece in dynamic test in the case of expansion ratio of 12; at engineering strain of (a) 0.04 , (b) 0.08 , (c) 0.12 , (d) 0.19 , (e) 0.32 , (f) 0.44 .

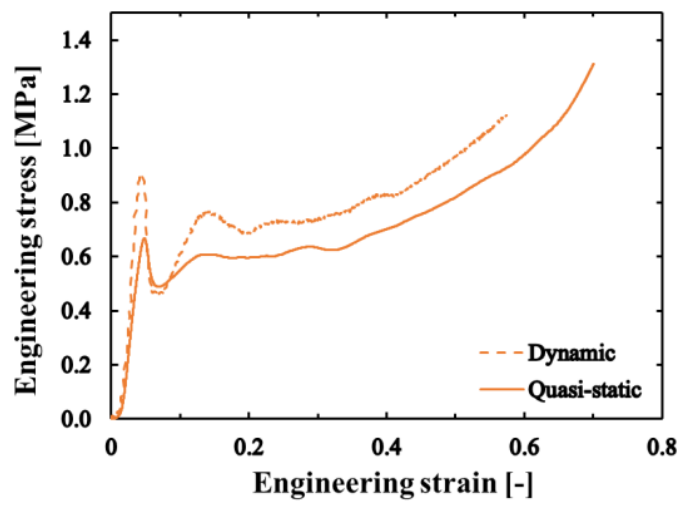

Fig. 5. The stress-strain curve in the case of expansion ratio of 12 .

The strain distribution obtained by DIC in the quasi-static and dynamic test is shown in Figs. 6 and 7, respectively. After the plastic collapse, it was confirmed that cell buckling was initiated around the middle in the height (Figs. 6(b) and 7(b)), then propagated to the whole specimen in both the quasi-static and dynamic tests. Although the stress value in the stressstrain curves was different between the quasi-static and the dynamic test, the deformation mode seemed to be the same in the test condition in this study.

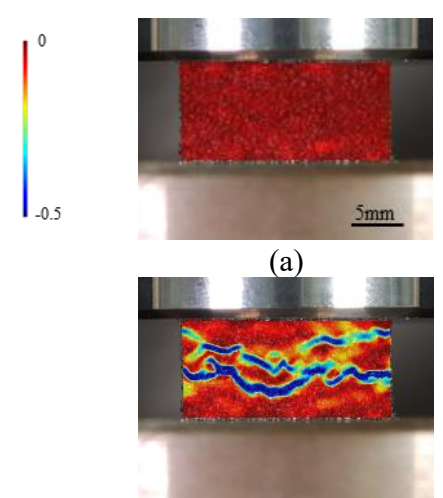

(d)

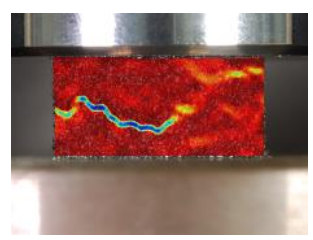

(b)

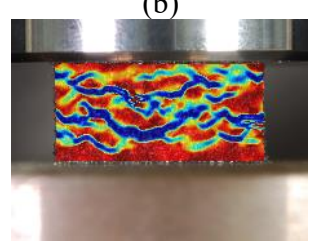

(e)

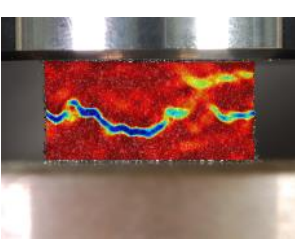

(c)

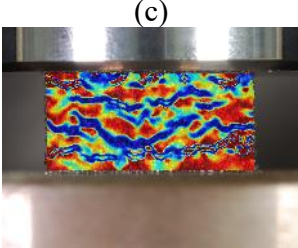

(f)

Fig. 6. Strain distribution in quasi-static test in the case of expansion ratio of 12; at engineering strain of (a) 0.04 , (b) 0.07 , (c) 0.10 , (d) 0.20 , (e) 0.30 , (f) 0.45 


$$
\left.\right|_{-0.5} ^{0}
$$

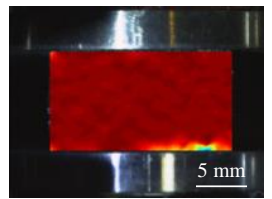

(a)

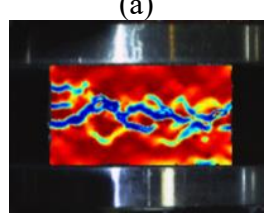

(d)

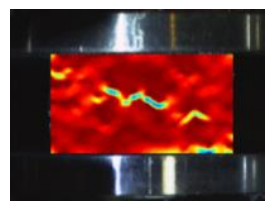

(b)

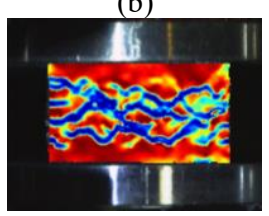

(e)

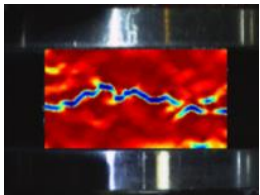

(c)

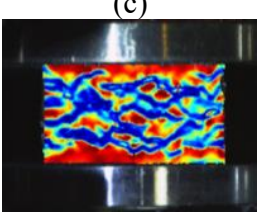

(f)

Fig. 7. Strain distribution in dynamic test in the case of expansion ratio of 12 ; at engineering strain of (a) 0.04 , (b) 0.08 , (c) 0.12 , (d) 0.19 , (e) 0.32 , (f) 0.44 .

From the obtained stress-strain curve, the amounts of absorbed energy were estimated with Eq. (1) and summarized in a graph (Fig. 8). It was confirmed that the amount of absorbed energy was increased with larger relative density (smaller expansion ratio) or with a higher strain rate. The strain rate dependence of the amount of absorbed energy may be due to the deformation mode in which the local buckling of the cells is dominant.

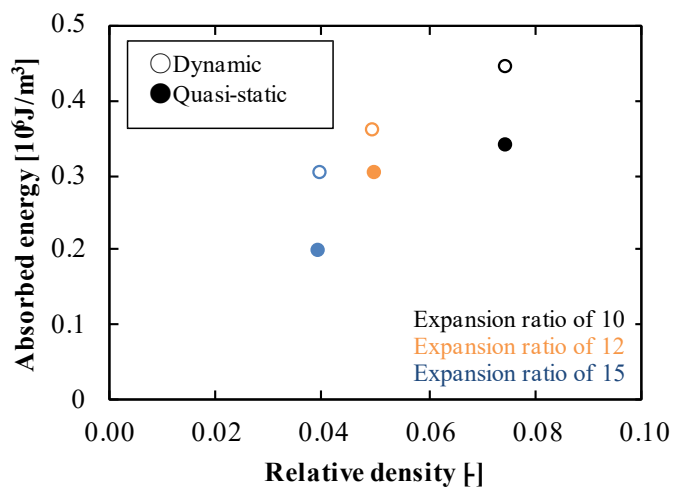

Fig. 8. Relationship between absorbed energy and relative density.

\section{Summary}

The compressive responses and energy absorption characteristics of polypropylene foamed plastic were evaluated. The stress-strain curves obtained from the quasi-static and dynamic compression test indicated that the plastic collapse of the test piece was induced by local buckling of cells. Also, it was confirmed that the plastic collapse stress was increased with a higher strain rate. This may because the buckling of the cells was the dominant deformation mode in the plastic collapse of the test piece. With the strain distribution obtained by DIC, it was confirmed that cell buckling was initiated around the middle in the height after the plastic collapse, then propagated to the whole specimen in both the quasi-static and dynamic tests. The amount of absorbed energy was increased with larger relative density (smaller expansion ratio) or with a higher strain rate. The strain rate dependence of the amount of absorbed energy may be due to the deformation mode in which the local buckling of the cells is dominant. 


\section{References}

1. L. J. Gibson, M. F. Ashby, Cellular Solids: Structure and Properties (Cambridge University Press, Cambridge, 1997)

2. N.J. Mills, C. Fitzgerald, A. Gilchrist, R. Verdejo, Composites Science and Technology, 63 (2003)

3. N. J. Mills, H. X. Zhu, J. Mech. Phys. Solids, 36 (1999)

4. S. Fushimi, T. Nagakura, A. Yonezu, Polymer Testing, 63 (2017)

5. Yongle Sun, B. Amirrasouli, S.B. Razavi, Q.M. Li, T. Lowe, P.J. Withers, Acta Mater., 110 (2016)

6. C. Barbier, P.M. Michaud, D. Baillis, J. Randrianalisoa, A. Combescure, Eur. J. Mech. A Solids, 45 (2014)

7. K. Tateyama, H. Yamada, N. Ogasawara, Polymer Testing, 52 (2016)

8. J. Blaber, B. Adair \& A. Antoniou, Experimental Mechanics, 55 (2015)

9. C. R. Calladine and R. W. English, Int. J. Mech. Sci. 26, 11/12 (1984) 